\title{
A PARALLEL CLUSTER LABELING METHOD FOR MONTE CARLO DYNAMICS
}

\author{
Mike Flanigan and Pablo Tamayo \\ Thinking Machines Corp. \\ 245 First. St. Cambridge, MA 02142 U.S.A
}

Received $(\ldots \ldots .$.
Revised $(\ldots \ldots .$.

ABSTRACT

\begin{abstract}
We present an algorithm for cluster dynamics to efficiently simulate large systems on MIMD parallel computers with large numbers of processing nodes. The method divides physical space into rectangular cells which are assigned to processing nodes and combines a serial procedure, by which clusters are labeled locally inside each cell, with a nearest neighbor relaxation process in which processing nodes exchange labels until a fixed point is reached. By controlling overhead and reducing inter-processor communication this method attains good performance and speed-up. The complexity and scaling properties of the algorithm are analyzed. The algorithm has been used to simulate large twodimensional Ising systems (up to $27808 \times 27808$ sites) with Swendsen-Wang dynamics. Typical updating times on the order of 82 nanosecs/site and efficiencies larger than $90 \%$ have been obtained using 256 processing nodes on the CM- 5 supercomputer.
\end{abstract}

Keywords: Ising Model, Cluster Labeling, Percolation, Monte Carlo Simulations, Accelerated Dynamics.

\section{Introduction}

Over the last five years, since the introduction of the Swendsen-Wang (SW) algorithm, ${ }^{1}$ cluster dynamics have been extended to a variety of models in Statistical Mechanics and Field Theory ${ }^{2}$. These new "accelerated dynamics" update percolation clusters instead of single spins producing faster decorrelation times and reduced critical slowing down ${ }^{3}$. In fact, the dynamic critical exponent $z$ is reduced significantly or even eliminated in some cases. This acceleration does not come for free; the price to pay is an increased computational complexity compared with standard Metropolis, heat bath or other local algorithms. The cluster labeling procedure requires a fair amount of non-local (unstructured) data movements that makes cluster algorithms intrinsically hard to vectorize or paralellize. It is important to develop efficient cluster labeling methods because they are used every time step inside the core of the simulations. One has to worry not only about their complexity and scaling properties but also about absolute execution times. One of the modern challenges of computational science is to find efficient algorithms to exploit the unprecedented computational power of today's massively parallel supercomputers. 
Cluster labeling on a lattice is also relevant for the analysis of structures obtained in many computer simulations of statistical systems: Ising and percolation clusters, ${ }^{4}$ nucleation droplets, polymers, crystals, fractal structures, and particle tracks in experimental high energy Physics. Cluster labeling is a special case of the more general problem of finding the connected components of a graph, ${ }^{5}$ which has applications in computer vision, image processing and network analysis, among others.

In this paper we propose a simple method for cluster labeling that can be used at the core of Monte Carlo simulations on $\mathrm{MIMD}^{i}$ parallel computers and in particular the CM-5 (Thinking Machines Corp.). The algorithm we will present is a general method for cluster finding in $n$-dimensional Euclidean lattices, but we will concentrate on the specific problem of cluster labeling for the Ising Model with Swendsen-Wang dynamics. Percolation bonds are defined between aligned spins with bond probability $p_{b o n d}=1-e^{-2 \beta}$, and the clusters of connected spins, the Coniglio-Klein ${ }^{6,7,8}$ percolation clusters, are flipped with $50 \%$ probability. At the critical point, where most of the interesting behavior occurs, the clusters span the system and information has to be propagated across the entire computational domain.

Fig. 1. Typical critical clusters in the two-dimensional Ising model. The lines show the partitioning of the system into 16 cells (processing nodes).

\footnotetext{
${ }^{i}$ Multiple Instruction Multiple Data
} 
Several methods to find connected components have been introduced in the Computer Science literature. There are general methods based on union-find, ${ }^{9,10}$ transitive closure ${ }^{11}$ vertex collapse ${ }^{11,12,13,14}$ and vector models ${ }^{15}$. We realized that most of these methods are not well suited to practical lattice Monte Carlo simulations on MIMD machines where one has to worry about absolute execution times over average configurations instead of worse cases. Most of these methods are designed for idealized PRAM ${ }^{i i}$ models and require extensive global communications, shared memory or data format transformations. Some other methods are more appropriate for SIMD ${ }^{i i i}$ fine-grained machines ${ }^{15,16,17,25,29}$. The choice of data partitioning is very important ${ }^{18,9}$. A number of MIMD cluster labeling algorithms, specifically designed for cluster Monte Carlo simulations have appeared in the literature, ${ }^{19,21}$ but have very limited speed-up and efficiency which limits their application to the simulation of large systems using large numbers of processing nodes. The algorithm with the best scaling properties appears to be the self-labeling method introduced by Baillie and Coddington ${ }^{21}$. Recently, Kertesz and Stauffer ${ }^{20}$ have used the strip geometric parallelization method to simulate large systems $\left(6400^{2}\right)$ on the Intel iPSC/860. These methods have greatly improved our knowledge of the behavior of large systems but still lack the required scaling properties to efficiently utilize hundreds or thousands of processing nodes. In this paper we propose a method appropriate for the simulation of large systems on large numbers of processing nodes which attains unprecedented speed-up and efficiency. The method is based on a rectangular domain decomposition strategy. Similar partitioning techniques have been used by Tucker, ${ }^{18}$ Embrechts et al, ${ }^{22}$, Baillie and Coddington ${ }^{21}$ and by D. Rapaport who has simulated extremely large systems $\left(640000^{2}\right)$ by sequentially loading one square subsystem at a time on an IBM RS $/ 6000^{23,20}$. In our scheme the clusters are first labeled locally inside each processing node and then labels are propagated across processing nodes by a relaxation process. In section two we describe the algorithm and discuss some of its properties. In section three we will analyze its time complexity and scaling properties. Numerical results are discussed in section four. Finally, section five contains conclusions.

\section{Description of the Algorithm}

Physical space is divided into rectangular cells in such way that each cell is assigned to one processing node (see Figure 1). The algorithm labels the clusters in two stages: first it finds all the clusters inside each processing node using a serial local algorithm, and then it performs a global relaxation process in which processing nodes exchange clusters labels with nearest neighbors until a fixed point is reached. The operations of the algorithm are shown in Figures 2 and 3. The procedure can be described as follows:

\footnotetext{
${ }^{i i}$ Parallel Random-Access Machine.

${ }^{i i i}$ Single Instruction Multiple Data.
} 
Fig. 2. (a) shows the initial state and connectivity of a sub-system on one processing node. (b) shows the local roots (in black) and the sites that point to them. (c) illustrates the data structures setup for the relaxation cycle.

\section{Procedure Cluster-Labeling:}

(i) Define connectivity for the sites: for Swendsen-Wang dynamics connectivity bonds are defined with probability $p_{\text {bond }}=1-e^{-2 \beta}$ between aligned spins.

(ii) In each processing node independently apply the serial algorithm to find clusters (Procedure Local). At boundary sites the off-node bonds are ignored. At the end all sites are labeled with their corresponding "local roots", which are then globalized ${ }^{v}$.

(iii) Iterate relaxation cycles, exchanging local root labels with neighboring processing nodes until there is no change in any node (Procedure Relax). At the end all sites get their final global label from their local roots.

(iv) Clusters are flipped with $50 \%$ probability and measurements of relevant quantities are accumulated (energy, magnetization etc.).

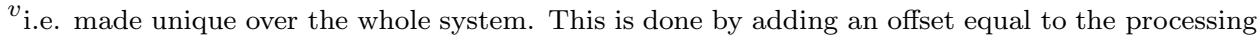
node number times the size of the local system $(n)$. For the Swendsen-Wang dynamics we also multiply the result by two and add a random bit - in this way we can "piggy-back" flipping information in the parity of the cluster label.
} 
Fig. 3. Processing nodes exchange local root labels with nearest neighbors. Minimum labels are found and local root labels are updated. The process is repeated until there are no more changes in local root labels.

The serial local algorithm we used has similarities to both Hoshen-Kopelman ${ }^{24}$ and union-find ${ }^{10}$ algorithms ${ }^{v i}$. This particular serial algorithm proved efficient for our approach but other serial algorithms can be used. It can be briefly described as follows:

\section{Procedure Local:}

(i) Assign a unique label to each site (e.g. if sites are stored in a one dimensional array then the label is the array index of the site itself).

(ii) For each site, follow the label paths for the site and each of its connected neighbors until their roots (sites pointing to themselves) are found. Then obtain the minimum label and set the site label, its root, and the roots of the connected neighbors to the minimum value.

(iii) To finish the labeling process a final collapse of trees is done by a pass over all sites setting each site to point to its corresponding root.

After the local labeling is completed (see Fig. 2b) a number of relaxation cycles are executed to label the clusters globally. The relaxation procedure consists of the

\footnotetext{
${ }^{v i}$ We also used some ideas from an earlier serial code developed by R. Giles, R. Brower and P. Tamayo at Boston University.
} 
following:

\section{Procedure Relax:}

(i) Execute a preparation step to set data structures: for each node boundary define a list of pointers to local roots (see Fig. 2c), one pointer per each bond crossing node boundaries.

(ii) Execute a sequence of relaxation cycles until all nodes detect no change in the labels (see Fig. 3):

(a) Each processing node interchanges boundary labels with the neighboring node in each direction. The labels are sent in a single block of data using synchronous message-passing calls.

(b) The local root labels are compared with the ones obtained from the neighbors and then set to the minimum values.

We find that even for large numbers of processing nodes the execution time is dominated by the local part. Originally we intended to implement a multigrid scheme for the relaxation part, as was used in ref. 25. This extension is straightforward and makes the algorithm more scalable but we have found that in practice it is not necessary given the sizes of coarse-grained MIMD machines available today. Even a large parallel computer with 1024 processing nodes forms relatively small lattices in 2 and 3 dimensions which would require only a few multigrid levels. Nearest-neighbor relaxation is very efficient for this problem at the scale of the processing nodes grid.

\section{Analysis: Complexity and Scaling}

Considering the fact that the cluster labeling algorithm operates at the core of equilibrium Monte Carlo simulations the analysis will focus on average case instead of worst case performance. The probability of obtaining a given configuration of clusters is determined by the Boltzmann weight of that particular configuration. The probability of observing the worst case is negligible. The execution times we will consider correspond to averages over equilibrium configurations generated in the Monte Carlo process. Furthermore, we will analyze the properties of the algorithm at the critical point for the Ising model.

In this section we will make a simplified model for the time complexity of the algorithm based on simple scaling arguments. The predictions of the model will be compared with experimental results in the next section. Similar analysis for the geometric parallelization method have been presented by Burkitt and Heermann ${ }^{19}$ and Jakobs and Gerling ${ }^{26}$.

In the following discussion, $N=L \times L$ is the total size of the system, $n=l \times l$ is the size for the subsystem in each processing node, and $p$ is the total number of processing nodes. Clearly the total number of sites is $N=n p$. In addition, $a$ and $b$ are constants. We start by considering the total time to perform the cluster 
labeling as consisting of two contributions: a "local time", the time spent by the serial algorithm inside each processing node, and a "relax time" which is the time spent in the global relaxation procedure until completion. The scaling of the local part is basically $O\left(n \log ^{*} n\right)$ where $\log ^{*} n$ is a very slow growing function ${ }^{v i i}$. In practice, as it is discussed in ref. 10, the value of $\log ^{*} n$ can be safely considered a constant and the serial time is basically $O(n)$,

$$
T_{\text {local }}=a l^{2}=a n .
$$

To compute the relaxation time we need to compute two contributions: $t_{\text {relax }}$, the time to do one relaxation cycle and $n_{\text {relax }}$, the number of relaxation cycles needed to complete the process. $t_{\text {relax }}$ is proportional to the size of the boundary between processing nodes, therefore, assuming the communication times grow linearly with message size, we have,

$$
t_{\text {relax }} \sim l \sim n^{1 / 2}
$$

If we consider relaxation as a nearest-neighbor propagation of labels, then $n_{\text {relax }}$ should be proportional to the maximum depth of the clusters embedded in the lattice. The maximum depth of the clusters is equivalent to the maximum shortest path joining two sites over the set of clusters: $\lambda_{\min }$; this length, also known as the chemical distance ${ }^{27}$, characterizes the way information is transmitted inside a cluster by step-by-step processes such as nearest neighbor label propagation. Typically for percolation clusters, $\lambda_{\min }$ scales with cluster linear size $r$ with a characteristic exponent $d_{\min }$,

$$
\lambda_{\min } \sim r^{d_{\min }},
$$

which implies that the number of relaxation cycles to label a given cluster will scale with $d_{\text {min }}$. Since the clusters can be as large as the entire system $(r=L)$, then $n_{\text {relax }}$ will be proportional to $L^{d_{\min }}$. However, in our case we have relaxation only at the scale of the processing nodes, not at the scale of single sites, and then the correct length to use in the scaling expression for $n_{\text {relax }}$ is the renormalized length $L / l=p^{1 / 2}$,

$$
n_{\text {relax }} \sim(L / l)^{d_{\text {min }}} \sim p^{d_{\text {min }} / 2} .
$$

The connectivity at lower scales is "integrated out" by the local procedure but we are assuming that the chemical distance of the resulting renormalized lattice still scales with the same exponent $d_{m i n}$. The value of $d_{m i n}$ for $2 \mathrm{~d}$ Coniglio-Klein clusters is reported to $\mathrm{be}^{28} 1.08 \pm 0.01$; consequently we expect $n_{\text {relax }}$ to grow

\footnotetext{
${ }^{v i i} \log ^{*} n$ is equal to the number of times the $\log$ function has to be applied recursively to the argument until it converges to one.
} 
slightly faster than the square root of the number of processing nodes ${ }^{v i i i}$. Now we can express the total relaxation time, $T_{\text {relax }}$, as

$$
T_{\text {relax }}=n_{\text {relax }} t_{\text {relax }}=b p^{d_{\min } / 2} n^{1 / 2},
$$

where we can see that $d_{\min }$ plays the role of a "computational" critical slowing down exponent for the algorithm. This is one of the cases in which a physical parameter of the simulated system, such as the chemical length exponent, determines its computational properties.

The total time for the parallel algorithm is then the sum of local plus relax times,

$$
T_{\text {parallel }}=T_{\text {local }}+T_{\text {relax }}=a n+b p^{d_{\min } / 2} n^{1 / 2} .
$$

If $n$ is large compared with $p$, and the communication to computation ratio $a / b$ is small, the scaling will be dominated by the local part. This can be seen more explicitly by calculating the speed-up function $S$, which is the ratio between the serial and parallel times,

$$
S=\frac{T_{\text {serial }}}{T_{\text {parallel }}}
$$

We will use as $T_{\text {serial }}$ the time of the local algorithm running on only one processing node. We can express $S$ as a function of $n$ and $p$ in the following way,

$$
S(n, p)=\frac{a n p}{a n+b p^{d_{m i n} / 2} n^{1 / 2}} .
$$

As expected the speed-up improves with large $n$ and gets worse as $p$ increases. Usually, the speed-up is computed as a function of $p$ for fixed system size $N$,

$$
S_{N}(p)=\frac{a N p}{a N+b p^{\left(d_{\min }+1\right) / 2} N^{1 / 2}} .
$$

The corresponding efficiency $E_{N}(p)=S_{N}(p) / p$ is

$$
E_{N}(p)=\frac{1}{1+\frac{b}{a}\left[p^{\left(d_{\min }+1\right)} N^{-1}\right]^{1 / 2}} .
$$

The efficiency decreases as the number of processing nodes increases because inter-processor communication times will eventually dominate. In practice it is important to make $b / a$ as small as possible. As we will see in the next section, this can be done effectively on the CM- 5 where $b / a \simeq 1$. It is interesting to notice that the efficiency is a universal function of $p^{\left(d_{\min }+1\right)} N^{-1}$. In terms of efficiency the only

${ }^{v i i i}$ The use of a multigrid or hierarchical scheme can make $n_{\text {relax }}$ scale as $\log ^{2} p$, see for example refs. 25 and 29 . 
Fig. 4. Local, relax and total times for $2 \mathrm{~d}$ SW dynamics as a function of $N^{1 / 2}$ for fixed $p=256$.

Fig. 5. Local, relax and total times for $2 \mathrm{~d}$ SW dynamics as a function of $p$ for fixed $n$. 
important parameter in the simulation is the value of $p^{\left(d_{m i n}+1\right)} N^{-1}$ : the larger $N$ is in relation to $p$ the better the algorithm will perform.

\section{Numerical Results}

We have implemented the algorithm using standard C language plus messagepassing calls (for inter-processor communication) using the CM-5 CMMD library ${ }^{\text {viii }}$. Detailed information about the CM-5 and its network architecture can be found in ref. 30 .

Fig. 6. The number of relaxation cycles as a function of the number of processing nodes for fixed $n=128^{2}$. The dot-dashed line gives the asymptotic slope predicted by $d_{\min } / 2$.

We have performed simulations of the 2d Ising model with Swendsen-Wang dynamics at the critical point and measured execution times for different values of $n$ and $p$, and total system sizes $N$ from $256^{2}$ to $27808^{2}$, using up to 256 processing nodes on CM-5 machines without vector units (SPARC node processors). Tables 1 and 2, and Figures 4 and 5 show times (local, relax, and total) for different system sizes and numbers of processing nodes. Measurements times (energy, magnetization, etc.) were not included in the timings. As we can see in Figures 4 and 5, local times dominate for large systems and we obtain good performance. Typical updating times are 314 nanosecs/site for a 64 node CM-5 and 82 nanosecs/site on a 256 node CM-5.

$\overline{v i i i}$ The program is about 600 lines of code and it will be available from the authors. 
Fig. 7. Total time as a function of inverse temperature $\beta$.

Fig. 8. Speed-up data for up to 256 processing nodes for different system sizes. 
Table 1. Timings for $2 \mathrm{~d}$ SW dynamics.

\begin{tabular}{cllll}
\hline \multicolumn{5}{c}{$p=64$} \\
\hline$N$ & local [secs] & relax [secs] & total [secs] & nanosecs/site \\
\hline $256^{2}$ & 0.019 & 0.010 & 0.029 & 442 \\
$512^{2}$ & 0.076 & 0.014 & 0.090 & 343 \\
$1024^{2}$ & 0.307 & 0.021 & 0.328 & 313 \\
$2048^{2}$ & 1.264 & 0.027 & 1.291 & 308 \\
$4096^{2}$ & 5.188 & 0.089 & 5.277 & 314 \\
$8192^{2}$ & 20.94 & 0.143 & 21.09 & 314 \\
\hline
\end{tabular}

Table 2. Timings for $2 \mathrm{~d}$ SW dynamics.

\begin{tabular}{cllll}
\hline \multicolumn{5}{c}{$p=256$} \\
\hline$N$ & local [secs] & relax [secs] & total [secs] & nanosecs/site \\
\hline $512^{2}$ & 0.020 & 0.025 & 0.045 & 172 \\
$1024^{2}$ & 0.079 & 0.027 & 0.106 & 101 \\
$2048^{2}$ & 0.318 & 0.035 & 0.353 & 84 \\
$4096^{2}$ & 1.277 & 0.078 & 1.355 & 81 \\
$8192^{2}$ & 5.232 & 0.159 & 5.391 & 80 \\
$16384^{2}$ & 21.27 & 0.341 & 21.61 & 81 \\
$27808^{2}$ & 62.87 & 0.566 & 63.44 & 82 \\
\hline
\end{tabular}

Simulating a $27808^{2}$ system requires about 15 Mbytes of local memory on a 256 node CM- 5 . This is about 5 bytes per site ( 4 bytes, one integer, for the label and one byte for spin and bond connectivity information). Using 32 Mbytes of memory systems as large as $40600^{2}$ can be simulated on 256 nodes. The local procedure is not particularly amenable to vectorization but the use of the parallelism provided by the 4 vector units on each CM-5 node, which support integer operations, will increase the speed of the local procedure significantly.

The scaling behavior of the measured times agrees well with the simple scaling model of the previous section (Equations 1-5): local times scale linearly with $n$, and relaxation times with $n^{1 / 2}$ (see Fig. 4). The number of relaxation cycles as a function of $p$ for fixed $n$ approaches the asymptotic slope $d_{m i n} / 2$ as can be seen in Fig. 6. From these data we obtain $b / a \simeq 1$. Figure 7 shows the total time as a function of temperature where the peak corresponds to the critical point.

The speed-up $S_{N}(p)$ as a function of the number of processing nodes for different system sizes $N$ is shown in Figure 8. Notice that for large system sizes the speed-up 
Fig. 9. Efficiency data for up to 256 processing nodes for different system sizes.

keeps increasing without saturation even for our maximum $p$ equal to 256 processing nodes. The algorithm has much better speed-up and efficiency than previous methods ${ }^{i}$. The $27808^{2}$ lattice is simulated with $92 \%$ efficiency. The functional form of these curves is given by Eq. 9. The efficiency $E_{N}(p)=S_{N}(p) / p$ as a function of $p$ is shown in Fig. 9. Simulations of large systems $\left(>2048^{2}\right)$ attain efficiencies higher than $90 \%$ even for up to 256 processing nodes. When these points are plotted as a function of $\left[p^{\left(d_{\min }+1\right)} N^{-1}\right]^{1 / 2}$ (see Fig. 10) they show the scaling described by Eq. 10: data for different system sizes collapses reasonably well to a universal curve. In this plot, as well as in the others, there are fluctuations in the timings that cause small deviations from the expected behavior. This is due to cache memory effects and non-linear behavior in the message-passing communications. We have not tried to take these effects into account in the scaling model because they are hard to estimate and will make the analysis unnecessarily complicated.

\section{Conclusions}

We have found that rectangular domain decomposition and a combination of a local method with global relaxation produces much better scaling properties than other methods. A careful choice of data structures, partitioning and communication strategies is fundamental to produce practical and efficient algorithms for Monte

${ }^{i}$ For example compare our Fig. 8 with Fig. 5 of ref. 19 (first paper) or Figures 4,5 and 6 of ref. 21. 
Fig. 10. Universal scaling of efficiency data plotted as a function of $\left[p^{\left(d_{\min }+1\right)} N^{-1}\right]^{1 / 2}$. The solid line is the predicted behavior given by Equation 10 (with $a / b=1$ ).

Carlo simulations. A simple scaling model for the complexity of the algorithm gives reasonable predictions for the observed times, speed-up and efficiencies.

Our algorithm running on a 256 node CM-5 is about 79 times faster than a Swendsen-Wang program ${ }^{32}$ on a Cray-XMP $(6.5 \mu$ secs/site), which clearly indicates that MIMD parallel computers, and the CM-5 in particular, can be used very effectively for this kind of computational problems.

It is interesting to notice that many of the most efficient cluster algorithms, SIMD and MIMD, employ relaxation or relaxation plus multigrid methods: Brower et $a l^{25}\left(1.5 \mu \mathrm{sec} / \mathrm{site}, 64 \mathrm{~K}\right.$ CM-2), Baillie and Coddington ${ }^{21,31}(1 \mu \mathrm{sec} / \mathrm{site}$, Symult 192 nodes and nCUBE-2 64 nodes), Apostolakis et al ${ }^{29,31}$ (6.5 $\mu$ secs/site, 16K CM2 ) and this work ( 82 nanosecs/site, CM-5 256 nodes). Other methods are also being improved. Recently, Apostolakis, Coddington and Marinari ${ }^{31}$ obtained speeds of $1.36 \mu \mathrm{secs} / \mathrm{site}$ on a $32 \mathrm{~K}$ CM-2 using a global get/send method. Kertesz and Stauffer ${ }^{20}$ attained speeds of about $0.5 \mu$ secs/site with their Intel iPSC/860 (8 nodes) implementation of the strip geometric parallelization method.

We hope our cluster labeling method will provide a useful tool for the study of large systems and the calculation of critical exponents, correlation functions etc, with unprecedented accuracy. It can be applied to simulations of Ising and Potts models, ${ }^{1,2}$ embedded dynamics simulations of Landau-Ginzburg and Heisenberg models ${ }^{2}$ virtual bond percolation dynamics ${ }^{33}$ and the study of static and dynamic 
properties of percolation clusters ${ }^{4,6}$.

\section{Acknowledgments}

We want to thank L. Tucker, C. Feynmann, D. Stauffer, G. Blelloch, A. Greenberg, R. Giles, R. Brower, W. Klein, N. Copty, B. Boghosian, G. Batrouni, R. Jones, M. Drumheller, G. Drescher, R. Frye, M. Best, P. Coddington, J. Apostolakis and E. Marinari, for interesting discussions about cluster methods and their applications. We want to thank one anonymous referee for all his valuable and helpful comments which motivated us to improve our program. We also thank the members of the CM-5 CMMD team for their technical advice and useful suggestions. We express our appreciation to C. Madsen, J. Mesirov, L. Tucker and J. Mucci for supporting this project. Some of the simulations were done on CM-5 machines at National Center for Supercomputer Applications (NCSA) and the Pittsburgh Supercomputer Center (PSC).

\section{References}

1. R. Swendsen and J. S. Wang, Phys. Rev. Lett., 58, (1987) 86.

2. R. Brower and P. Tamayo, Phys. Rev. Lett., 62, (1989) 1087; R. Brower and S. Huang, Phys. Rev. D 41, (1990) 708; U. Wolff, Phys. Rev. Lett. 60, (1988) 1461; R. Edwards and A. Sokal, Phys. Rev. D 38, (1988) 2009; W. Klein, T. Ray and P. Tamayo, Phys. Rev. Lett., 62, (1989) 163; P. Tamayo, R. C. Brower and W. Klein, J. of Stat. Phys., 58, (1990) 1083;

3. P. C. Hohenberg and B. Halperin, Rev. of Mod. Phys., 49, (1977) 435.

4. D. Stauffer and A. Aharony, Introduction to Percolation Theory 2nd. ed., Taylor and Francis, London (1992).

5. T. H. Cormen, C. E. Leiserson and R. L. Rivest, Introduction to Algorithms, MIT Press, (1990); D. Knuth, The Art of Computer Programming, vol 3, Addison-Wesley (1973).

6. A. Coniglio and W. Klein, J. Phys. A, 13, (1980) 2775.

7. D. Stauffer, Physica A 171, (1991) 471; D. Stauffer and J. Kertesz, Physica A 177, (1991) 381; E. N. Miranda, Physica A 175, (1991) 235 , 179, (1991) 340. E. N. Miranda, Physica A 175, (1991) 229.

8. A. Coniglio, Phys. Rev. Lett., 62, (1989) 3054.

9. J. Woo and S. Sahni, Jour. of Supercomputing 3, (1989) 209.

10. E. Horowitz and S. Sahni, Fundamentals of Computers Algorithms, Potomac, Md. Computer Science Press, 1978.

11. M. J. Quinn and N. Deo, Computing Surveys, Vol. 16, No 3, September 1984.

12. Y. Shiloach and U. Vishkin, Jour. of Algorithms 3, (1982) 57.

13. U. Vishkin, Discrete Applied Mathematics 9, (1984) 197.

14. P. S. Gopalakrishnan, I. V. Ramakrishnan and L. N. Kanal, 1985 IEEE, Int. Conf. on Parallel Processing.

15. G. H. Blelloch, Vector Models for Data-Parallel Computing, MIT Press, 1990.

16. R. Cypher, J. L. C. Sanz and L. Snyder, Journal of Algorithms 10, (1989) 140.

17. W. Lim, A. Agrawal and L. Nekludova, Thinking Machines Tech. Report NA86-2.

18. L. W. Tucker, Proc. IEEE Conference on Computer Vision and Pattern Recognition, June 1986, Miami, Florida. 
19. A. N. Burkitt and D. W. Heermann, Comp. Phys. Comm. 54, (1989) 210; D. W. Heermann and A. N. Burkitt, Parallel Algorithms in Computational Science, Springer Verlag, Heidelberg 1991.

20. J. Kertesz and D. Stauffer, private communication.

21. C. F. Baillie and P. D. Coddington, Concurrency: Practice and Experience 3(2), (1991) 129.

22. H. Embrechts, D. Roose and P. Wambacq, Hypercube and Distributed Computers, F. Andre and J. P. Verjus eds. Elsevier Science Pubs. B. V. (North-Holland) 1989.

23. D. C. Rapaport, J. Phys. A 18, (1985) L175.

24. J. Hoshen and R. Kopelman, Phys. Rev. B, 14, (1976) 3438.

25. R. C. Brower, P. Tamayo and B. York, Jour. of Stat. Phys. 63, (1991) 73.

26. A. Jakobs and R. Gerling, Physica A 180, (1992) 407.

27. H. J. Herrmann and H. E. Stanley, J. Phys. A 21 (1988) L829.

28. E. N. Miranda, Physica A 175, (1991) 229.

29. J. Apostolakis, P. Coddington and E. Marinari, Europhys. Lett. 17(3) (1992) 198;

30. C. H. Leiserson et al, The Network Architecture of the Connection Machine CM-5, The 1992 Symposium on Parallel Algorithms and Architectures, San Diego, CA 1992; The Connection Machine CM-5 Technical Summary, October 1991. Thinking Machines Corp. Cambridge, MA.

31. P. Coddington, J. Apostolakis and E. Marinari, private communication.

32. U. Wolff, Phys. Lett. B 228, (1989) 379 .

33. R. Brower and P. Tamayo, Virtual Bond Percolation for Ising Cluster Dynamics, Thinking Machines Tech. Report TMC 231 (1992); to be published in Physica A. 